\title{
Photodynamic Therapy: A Novel Ally for Surgical Endodontic Treatment? Case Report
}

\author{
Bruna Paloma de Oliveira ${ }^{1 *}$, Andréa Cruz Câmara ${ }^{1}$, Glauco dos Santos Ferreira ${ }^{2}$, Sebastião Pedro Santos \\ Neto $^{2}$, Patrícia Fernandes Cassimiro da Silva ${ }^{1}$, Carlos Menezes Aguiar ${ }^{1}$ \\ ${ }^{1}$ Department of Prosthodontics and Oral and Maxillofacial Surgery, school of dentistry, Federal University of Pernambuco, \\ Recife, Pernambuco, Brazil \\ ${ }^{2}$ Center for Post-Graduation in Dentistry, Seven Lagoons College, Recife, Pernambuco, Brazil
}

\author{
*Correspondence to \\ Bruna Paloma de Oliveira, DDS \\ MSc; Department of Prosthodontics \\ and Oral and Maxillofacial \\ Surgery, school of dentistry, Federal \\ University of Pernambuco, Av. \\ Prof. Moraes Rego s/n, Cidade \\ Universitária, Recife, PE Brazil \\ 50670901 \\ Tel: (+55) 81992853170 ; \\ Email: bruna_paloma@msn.com
}

Published online 17 September 2018

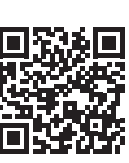

\begin{abstract}
Introduction: Photodynamic therapy (PDT) has emerged as a powerful tool for conventional endodontic treatment, capable of eradicating microorganisms present in endodontic infections. Despite this, the use of PDT in the surgical endodontic treatment is not well-known.

Case Report: This paper describes the case of a patient with asymptomatic apical periodontitis in a mandibular incisor submitted to surgical endodontic treatment associated with PDT. After conventional procedures, photosensitizer methylene blue $(0.01 \%)$ was applied to the retrograde cavity for 3 minutes, followed by irradiation with diode laser $(100 \mathrm{~mW}$ and $660 \mathrm{~nm})$ for 3 minutes. Six months after the procedure, the patient was asymptomatic, and the radiographic examination showed healthy periradicular tissues.

Conclusion: The association of PDT with surgical endodontic treatment was effective, suggesting that this therapy may provide additional benefits to patients when compared to the conventional surgical technique.

Keywords: Endodontics; Photodynamic therapy; Periradicular surgery.
\end{abstract}

\section{Introduction}

Surgical endodontic treatment is an option often suggested when it would be difficult or impossible to clean and shape the root canal system through a nonsurgical approach, or when a nonsurgical retreatment is unlikely to improve the previous results. ${ }^{1}$ One of the main objectives of periradicular surgery is to prevent the invasion of bacteria and their by-products from the root canal system into the periradicular tissues. ${ }^{2}$

In recent years, photodynamic therapy (PDT) has been shown to be a promising antimicrobial therapy capable of eliminating microorganisms presenting in endodontic infections. ${ }^{3,4}$ This treatment is based on the interaction of three components: a photosensitizer dye, a light source and oxygen. The mechanism of action of PDT occurs when the photosensitizer absorbs photons from the light source, and their electrons go into an excited state. Upon returning to its basic state, the photosensitizer transfers energy to a substrate, such as oxygen, forming free radicals of high cytotoxicity, which can cause the death of microorganisms. ${ }^{5}$

Several in vitro ${ }^{4}$ and in vivo ${ }^{6}$ studies have demonstrated that PDT is presented as an important antimicrobial tool for conventional endodontic treatment. Nevertheless, to date, only a few investigations ${ }^{7}$ have evaluated the use of this therapy in surgical endodontic treatment.

Thus, the purpose of this paper was to describe the case of a patient submitted to surgical endodontic treatment associated with PDT.

\section{Case Report}

A 24-year-old healthy male patient was referred for examination of tooth 42 . The access cavity of this tooth had been previously initiated by his general dentist. The patient reported no history of pain or swelling in this tooth, but gave a history of trauma to the anterior mental region a few years before. A clinical examination revealed no sinus tract or discolored crown. A periapical radiographic examination revealed the presence of a circumscribed radiolucent image in the periapical region. Additionally, the volume of the pulp cavity was greatly reduced, hampering visualization of the root canal (Figure 1).

Based on the clinical and radiographic characteristics, asymptomatic apical periodontitis and pulp canal obliteration was diagnosed. The clinical situation and

Please cite this article as follows: Oliveira BP, Câmara AC, Ferreira GDS, Neto SPS, Silva PFC, Aguiar CM. Photodynamic therapy: a novel ally for surgical endodontic treatment? Case report . J Lasers Med Sci. 2018;9(4):288-290. doi:10.15171/jlms.2018.51. 


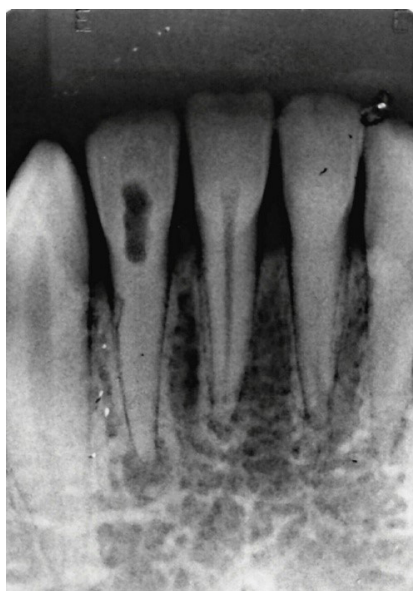

Figure 1. Preoperative Periapical Radiograph.

treatment options were debated with the patient, who accepted the attempt to perform a nonsurgical endodontic treatment as his first choice.

The informed consent was obtained, and after proper anesthesia and rubber dam isolation, the attempt to locate the root canal was initiated. However, due to the reduced volume of the pulp cavity, the root canal was not located, leading to the change of the initial treatment plan, which was modified by surgical endodontic treatment.

In a subsequent session, under infiltration anesthesia, a mucoperiosteal trapezoidal flap was raised. The osteotomy was performed with a carbide round bur, followed by curettage of the granulation tissue, and about 2-3 mm of the root apex was sectioned with a diamond cylindrical bur at high speed under irrigation with a sterile saline solution. Then, the retrograde cavity was prepared with an ultrasonic retro-tip (P1; Helse, Santa Rosa do Viterbo, SP, Brazil) (Figure 2a).

After the conventional procedures, the retrograde cavity, as well as, the surgical cavity received an aqueous solution of $0.01 \%$ methylene blue (Chimiolux; Hypofarma, Belo Horizonte, MG, Brazil) for three minutes (Figure 2b), and, subsequently, were irradiated with a diode red laser (Whitening Lase II; DMC, São Carlos, SP, Brazil; diameter tip $=30 \mathrm{~mm}$ ) for three minutes (wavelength $=660 \mathrm{~nm}$, power $=100 \mathrm{~mW}$, energy $=18 \mathrm{~J}$, energy density $=640 \mathrm{~J} /$ $\mathrm{cm}^{2}$, continuous emission mode) ${ }^{8}$ (Figure $2 \mathrm{c}$ ).

After PDT, the surgical cavity was irrigated with a saline solution, the retrograde cavity was filled with a mineral trioxide aggregate (MTA Angelus; Londrina, PR, Brazil) (Figure 2d), and the bone defect filled with calcium sulfate (Lumina-Set; Critéria, São Carlos, SP, Brazil) (Figure 2e). Finally, the flap was repositioned and sutured (Figure 2f). A periapical radiograph was performed immediately after the surgical procedure (Figure 3a).

The sutures were removed one week later, and the patient did not report any episodes of postoperative pain or discomfort. A regular follow-up was performed (Figure $3 \mathrm{~b}$ and $\mathrm{c}$ ), and after six months the radiographic

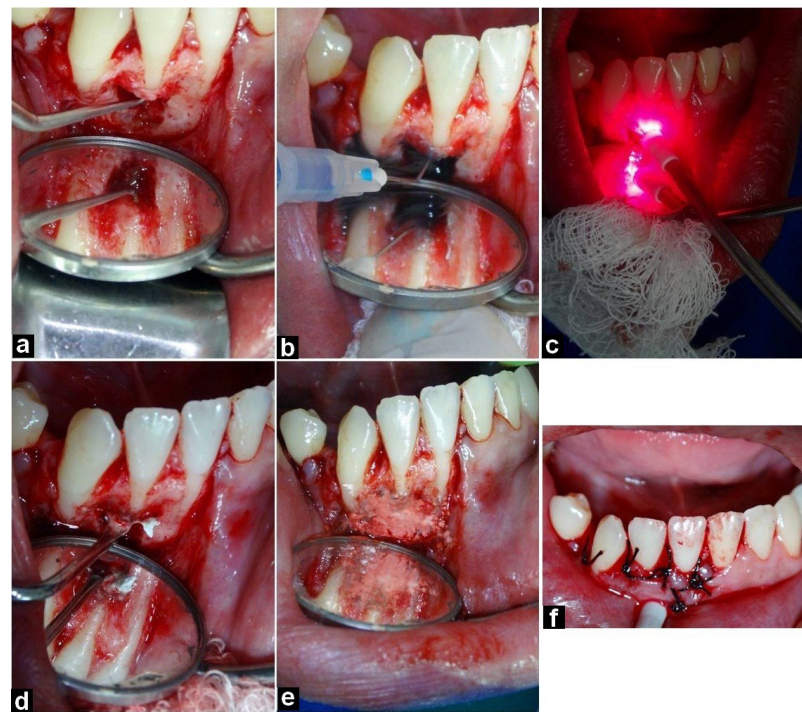

Figure 2. (a) Retrograde Cavity Preparation Using Ultrasonic Retro-Tip. (b) Application of The Methylene Blue Solution. (c) Irradiation With The Diode Laser. (d) Retrograde Cavity Filling With Mineral Trioxide Aggregate. (E) Bone Defect Filling With Calcium Sulfate. (f) Flap Sutures.

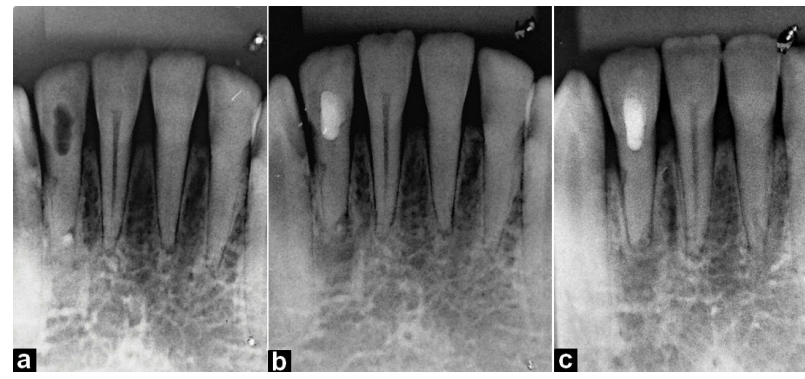

Figure 3. Follow-up Radiographic Images (a) Immediately After Surgery, (b) After 2 Months, (c) After 6 Months.

examination revealed bone formation and restructuring of the periapical tissues. No sinus tract or any signs of infection was verified.

\section{Discussion}

PDT has several attributes that make it an excellent tool to eliminate endodontic pathogens, such as: it covers a broad spectrum of action (bacteria, fungi, virus and protozoa), does not induce microbial resistance, eradicates pathogens in biofilms and inactivates endotoxins. Furthermore, this treatment is safe for the periapical tissues, easy to apply, painless, and inexpensive when compared to highintensity laser. ${ }^{3}$

Despite these diverse properties, the use of PDT associated with periradicular surgery is still scarcely mentioned in the literature. It is known that due to the polymicrobial contamination present in cases of periapical lesions, conventional surgical procedures alone may not be able to eradicate the existing infection completely. ${ }^{9}$ Thus; the use of a complementary antimicrobial therapy could be 
an alternative to enhance the decontamination process during periradicular surgery.

The study of Garcez et $\mathrm{al}^{7}$ demonstrated that the use of PDT in periradicular surgeries was able to provide a significant reduction of the microbial load present in the apical root dentin compared to the conventional technique performed without the use of PDT.

In the present report, six months after the procedure, the patient was asymptomatic, and the radiographic examination showed bone formation and restructuring of the periapical tissues of the treated tooth.

Besides the optimization of the antimicrobial action, other properties related to the use of PDT may have contributed to the effectiveness of this case. One of these properties refers to the use of the diode laser. It is known that the use of the laser in the red spectrum performs a biostimulatory action on the treated region, providing faster bone repair and tissue healing, besides reducing postoperative discomfort. ${ }^{10}$

It is important to reinforce the concept that the protocol for performing PDT associated with periradicular surgery still requires more scientific evidence. The execution of more clinical studies is necessary to allow the establishment of the ideal parameters for the application of this therapy, such as the energy dosage, photosensitizer concentration, and preirradiation time required.

In conclusion, the association of PDT to surgical endodontic treatment was effective in the present case, suggesting that the use of this therapy may provide additional benefits to patients than the conventional surgical technique.

\section{Ethical Considerations}

Ethical principles for medical research have been applied in the process of the research. Also, this study was submitted to and approved by the ethics committee of the Federal University of Pernambuco, Recife, Pernambuco, Brazil.

\section{Conflict of Interests}

The authors declare that there is no conflict of interest regarding the publication of this paper.

\section{References}

1. Nair PN. Pathogenesis of apical periodontitis and the causes of endodontic failures. Crit Rev Oral Biol Med. 2004;15:348-381.

2. Tsesis I, Rosen E, Schwartz-Arad D, Fuss Z. Retrospective evaluation of surgical endodontic treatment: traditional versus modern technique. J Endod. 2006;32:412-416. doi: 10.1016/j.joen.2005.10.051

3. de Oliveira BP, Aguiar CM, Câmara AC. Photodynamic therapy in combating the causative microorganisms from endodontic infections. Eur J Dent. 2014;8:424-430. doi: 10.4103/1305-7456.137662

4. de Oliveira BP, Aguiar CM, Câmara AC, de Albuquerque MM, Correia AC, Soares MF. The efficacy of photodynamic therapy and sodium hypochlorite in root canal disinfection by a single-file instrumentation technique. Photodiagnosis Photodyn Ther. 2015;12:436-443. doi: 10.1016/j. pdpdt.2015.05.004

5. Dai T, Huang YY, Hamblin MR. Photodynamic therapy for localized infections - State of the art. Photodiagnosis Photodyn Ther. 2009;6:170-188. doi: 10.1016/j. pdpdt.2009.10.008

6. Jurič IB, Plečko V, Pandurić DG, Anić I. The antimicrobial effectiveness of photodynamic therapy used as an addition to the conventional endodontic re-treatment: a clinical study. Photodiagnosis Photodyn Ther. 2014;11:549-55. doi: 10.1016/j.pdpdt.2014.10.004

7. Garcez AS, Arantes-Neto JG, Sellera DP, Fregnani ER. Effects of antimicrobial photodynamic therapy and surgical endodontic treatment on the bacterial load reduction and periapical lesion healing. Three years follow up. Photodiagnosis Photodyn Ther. 2015;12:575-580. doi: 10.1016/j.pdpdt.2015.06.002

8. Oliveira BP, Lins CC, Diniz FA, Melo LL, Castro CM. In Vitro antimicrobial photoinactivation with methylene blue in different microorganisms. Braz J of Oral Sci. 2014;13:5357.

9. Siqueira JF Jr, Rôças IN. Clinical implications and microbiology of bacterial persistence after treatment procedures. J Endod. 2008;34:1291-13013. doi: 10.1016/j. joen.2008.07.028

10. Yoshida T, Yamaguchi M, Utsunomiya T, Kato M, Arai Y, Kaneda T, et al. Low-energy laser irradiation accelerates the velocity of tooth movement via stimulation of the alveolar bone remodeling. Orthod Craniofac Res. 2009;12:289-298. doi: 10.1111/j.1601-6343.2009.01464.x 